\title{
MODELO DE FORMULACIÓN DE RACIONES AL MÍNIMO COSTO PARA GANADO DE CARNE BASADO EN EL SISTEMA NRC 2000
}

\author{
LEAST COST BEEF CATTLE RATION FORMULATION MODEL BASED ON \\ NRC 2000 SYSTEM
}

Soto, C. ${ }^{1}$ y Reinoso, V. ${ }^{1}$

${ }^{1}$ Actividad privada. Artigas. Uruguay. srvet@adinet.com.uy

\section{Palabras ClaVe ADICIONALES}

Programación lineal entera mixta. Pastoreo. Suplementación.

\section{RESUMEN}

Los requerimientos nutricionales del ganado de carne descrito por el NRC (2000) se establecen mediante ecuaciones, siendo algunas de ellas no lineales o no aditivas lo cual dificulta su incorporación a modelos de programación lineal para formular raciones al mínimo costo. El presente trabajo describe un modelo de programación lineal entero mixto para formular dietas de mínimo costo que incorpora las recomendaciones del Modelo Nivel Uno del NRC (2000). La falta de aditividad o linealidad de algunas funciones fueron superadas empleando restricciones alternativas modeladas con variables binarias. El modelo considera factores ambientales que modifican los requerimientos de los animales y los efectos de la dieta que afectan la eficiencia de síntesis de proteína microbiana a nivel ruminal y el consumo potencial voluntario de alimentos. Se consideran los requerimientos de energía, proteína metabolizable, calcio, fósforo y fibra efectiva. Se acota el consumo de pastura al consumo voluntario de la misma y el consumo de suplemento al máximo nivel de suplementación permitido. Se presenta un ejemplo de aplicación del modelo y se describen posibles ampliaciones.

\section{SUMMARY}

Nutritional requirements of beef cattle described by NRC (2000) are determinated by equations, some of which are nonlinear or non additive which makes it difficult for modelling with linear programming. This paper describes a model of mixed integer linear programming to formulate

Recibido: 13-6-11. Aceptado: 7-11-11.

\section{Additional KeYWORDS}

Mixed integer linear programming. Grazing. Supplementation.

least-cost diet that incorporates the recommendations of Model Level One of NRC (2000). The lack of additivity or linearity of some functions were overcome using alternatives restrictions modelling with binary variables. The model considers environmental factors that modify the requirements of the animals and the effects of the diet that affects the efficiency of microbial protein synthesis in the rumen and the level of voluntary feed intake. This model considers the requirements of energy, metabolizable protein, calcium, phosphorus and effective fiber. The intake of pasture is limited by the potential intake of pasture at grazing and the intake of supplement is limited by the maximum level of supplementation permitted. This paper shows an example of application of the model and describes possible extensions.

\section{INTRODUCCIÓN}

En Uruguay la producción ganadera se realiza casi exclusivamente a pastoreo con baja incorporación de suplementos, implementándose la suplementación en forma estructural básicamente en aquellos sistemas más intensivos, fundamentalmente en el período crítico invernal donde la producción de forraje disminuye marcadamente.

La pastura es el alimento de menor costo por unidad de nutrientes, por lo tanto, para un determinado nivel de producción es indispensable minimizar el costo total de la suplementación (Soto y Reinoso, 2004). 


\section{SOTOYREINOSO}

Desde que Waugh (1951) publicó su artículo para formular raciones al mínimo costo hasta la actualidad, la programación lineal (PL) se ha convertido en una herramienta insustituible en este campo. Los principios y métodos de solución (Hiller y Lieberman, 2002; Taha, 1998) así como las aplicaciones de la PL a la producción y a la nutrición animal (Black y Hlubick, 1980; Barnard y Nix, 1984; Beneke y Winterboer, 1973; Maroto et al., 1997) han sido descritos detalladamente en otros trabajos.

Numerosos sistemas de alimentación han sido modelados con PL (Tedeschi et al., 2000; Rotzet al., 1999; O'Connor et al., 1989; Black y Hlubick, 1980; Brokken, 1971) con el fin de poder formular dietas de mínimo costo.

La mayoría de los nutricionistas de Uruguay ajustan las dietas en ganado de carne mediante las recomendaciones más recientes del NRC (2000) lo cual dificulta la formulación de raciones de mínimo costo ya que estos modelos incorporan numerosas funciones no lineales y no aditivas.

El propósito de este trabajo es incorporar el Modelo Nivel Uno del NRC (2000) a un modelo de PL entero mixto para formular raciones al mínimo costo para ganado de carne en crecimiento y a pastoreo. Además se presenta un ejemplo de aplicación del modelo.

\section{MATERIAL Y MÉTODOS}

EINRC (2000) estima los requerimientos nutricionales teniendo en cuenta variaciones en el peso vivo, el tipo de ganado, el nivel de producción de leche y las condiciones ambientales, dividiendo al modelo de predicción en dos niveles, el nivel uno, más sencillo, de aplicación en aquellos casos en que la información sobre la composición de los alimentos es parcial (caso de Uruguay) y el nivel dos, más complejo que brinda información adicional sobre la utilización ruminal de carbohidratos y proteínas, y el aporte y requerimientos de aminoácidos al organismo. En muchos aspectos de ambos niveles del NRC (2000) la dieta y los requerimientos del animal son interactivos (dependiente uno del otro), por ejemplo el incremento calórico debido al estrés por frío; la eficiencia de utilización de la energía metabolizable (EM) para mantenimiento, crecimiento y lactación; la producción ruminal de proteína microbiana; el consumo potencial de materia seca, etc.

La aditividad (ausencia de dependencia de una variable de otra) y la proporcionalidad (linealidad) son algunos de los requisitos indispensables en los modelos de PL (Hiller y Lieberman, 2002; Taha, 1998). La dificultad de incorporar el nivel uno del modelo del NRC del 2000 a un modelo de PL consiste básicamente en cuatro aspectos, dado que dicho modelo asume que:

- La EM se emplea con diferente eficiencia para mantenimiento y crecimiento, dependiendo dicha eficiencia de la densidad energética de la dieta, por lo tanto los requerimientos y aportes de ENm y ENg no son aditivos.

- El consumo potencial de materia seca depende del contenido de ENm de la dieta y se estima mediante una función no lineal.

- El incremento de los requerimientos de mantenimiento por estrés por frío depende de la eficiencia de utilización de la EM de la dieta y del consumo total de EM.

- La eficiencia de síntesis de proteína microbiana depende del $\mathrm{pH}$ ruminal, el cual se relaciona con el contenido de eNDF de la dieta.

En el presente trabajo los problemas de aditividad y linealidad que presenta el modelo nivel uno del NRC (2000) para formular raciones al mínimo costo con PL fueron levantados empleando un modelo de PL entero mixto (PLEM), siendo la totalidad de las variables enteras binarias ( 0 o 1$)$. En todos los casos se trató de modelar empleando la notación original del NRC (2000). En las tablas I y II se listan las variables de decisión y las constantes paramétricas empleadas en el modelo de PLEM. 


\section{FunCIÓN OBJETIVO(FO)}

El objetivo en la formulación de raciones al mínimo costo es encontrar aquella combinación de alimentos que respetando las restricciones impuestas minimicen el costo total de la dieta $(Z)$.

$$
\text { FO) Minimizar } Z=P s{ }^{*} \mathrm{Cp} \$+\Sigma X_{j}{ }^{*} C_{j} \$
$$

\section{RESTRICCIONES}

\section{Consumo de materia seca}

La suma del consumo de pastura con suplementación (Ps) y de suplemento (Supl) es transferido al consumo total de materia seca (DMI). El consumo de pastura es acotado al consumo voluntario de la misma en pastoreo $(\mathrm{pI})$, mientras que el consumo de suplemento no puede superar el máximo permitido(Max_CMS_R).

R1) $P s+$ Supl - DMI $=0$

R2) $\mathrm{Ps} \leq \mathrm{pl}$
R3) $\Sigma X_{j}-$ Supl $=0$

R4) Supl $\leq$ Max_CMS_R

El consumo de pastura sin suplementación ( $\mathrm{pI}$ ) se determina mediante las ecuaciones usuales del NRC (2000, pág. 118 y 119). En general en Uruguay el nivel de suplementación en ganado de carne suele ser bajo (Orcasberro, 1994), niveles elevados de suplementación suelen afectar negativamente el consumo y la digestibilidad del forraje ocasionando una sustitución de forraje por concentrado (Moore et al., 1999; Caton y Dhuyvetter, 1997; Dixon y Stockdale, 1999; Bargo et al., 2003). Existen modelos de PL que incorporan explícitamente la tasa de sustitución de forraje por suplemento en la formulación de dietas (McCalletal., 1999; Soto y Reinoso, 2004). ElNRC (2000) no menciona corrección alguna en el consumo de pastura por efecto de la interacción suplemento-pastura, quedaría implícito en la ecuación que estima el

Tabla I. Variables de decisión del modelo. (Decision variables of model).

Ps= consumo de pastura (dieta base), kg MS/día.

$\mathrm{X}_{\mathrm{i}}=$ cantidad del j-esimo suplemento, $\mathrm{kg}$ MS/día.

Supl= consumo total de suplemento, $\mathrm{kg} \mathrm{MS/día.}$

$\mathrm{DMI}=$ consumo total de materia seca, $\mathrm{kg} \mathrm{MS/día.}$

MPfeed= proteína metabolizable aportada por la dieta, $\mathrm{kg} / \mathrm{día}$.

MPbact= producción proteína metabolizable microbiana, kg/día.

$\mathrm{MCP}=$ producción proteína bruta microbiana, $\mathrm{kg} / \mathrm{día}$.

$\mathrm{MEI}=$ consumo de EM, Mcal/día.

TDN_cons= consumo de TDN, kg/día.

UIP_cons= consumo de proteína no degradable en rumen, $\mathrm{kg} / \mathrm{día}$.

DIP_cons= consumo de proteína degradable en rumen, kg/día.

eNDF_cons= consumo de eNDF, kg/día.

Ca_cons $=$ consumo de calcio, $\mathrm{kg} / \mathrm{día}$.

$\mathrm{P} \_$cons $=$consumo de fósforo, $\mathrm{kg} / \mathrm{día}$.

EM_req= requerimientos totales de EM, Mcal/día.

$\mathrm{EM} \bar{m}=$ requerimientos de EM para mantenimiento, Mcal/día.

$\mathrm{EMg}=$ requerimientos de EM para crecimiento, Mcal/día.

EMmact= requerimientos de EM por actividad física del pastoreo, Mcal/día.

EMhs= requerimientos de EM por estrés por calor, Mcal/día.

EMcs= requerimientos de EM por estrés por frío, Mcal/día.

$y_{i}=i$-esima variable binaria auxiliar (parametrización del la EM de la dieta).

$\rho_{\mathrm{i}}=$ i-esima variable binaria auxiliar (parametrización del eNDF de la dieta). 


\section{SOTOYREINOSO}

Tabla II. Constantes del modelo. (Constants of model).

$\mathrm{Cp} \$=$ costo de la pastura, U\$S/kg MS.

$\mathrm{Cj} \$=$ costo del $\mathrm{j}$-esimo suplemento, U\$S/kg MS.

$\mathrm{pl}=$ consumo a pastoreo sin suplementación, $\mathrm{kg} \mathrm{MS/día.}$

Max_CMS_R= máximo consumo de suplemento permitido, $\mathrm{kg} \mathrm{MS/día.}$

$\mathrm{EM}_{0}=$ contenido de $\mathrm{EM}$ de la pastura, Mcal $/ \mathrm{kg} \mathrm{MS}$.

$\mathrm{EMj}=$ contenido de EM del j-esimo suplemento, Mcal/kg MS.

$\mathrm{TDN}_{0}=$ contenido de TDN de la pastura, $\mathrm{kg} / \mathrm{kg} \mathrm{MS}$.

TDNj= contenido de TDN del j-esimo suplemento, $\mathrm{kg} / \mathrm{kg} \mathrm{MS}$.

$\mathrm{UIP}_{0}=$ contenido de UIP de la pastura, $\mathrm{kg} / \mathrm{kg}$ MS.

UIPj= contenido de UIP del $\mathrm{j}$-esimo suplemento, $\mathrm{kg} / \mathrm{kg} \mathrm{MS}$.

DIP $_{0}=$ contenido de DIP de la pastura, $\mathrm{kg} / \mathrm{kg} \mathrm{MS}$.

DIPj= contenido de DIP del j-esimo suplemento, $\mathrm{kg} / \mathrm{kg} \mathrm{MS}$.

eNDF $_{0}=$ contenido de eNDF de la pastura, $\mathrm{kg} / \mathrm{kg} \mathrm{MS}$.

eNDFj= contenido de eNDF del j-esimo suplemento, $\mathrm{kg} / \mathrm{kg} \mathrm{MS}$.

$\mathrm{Ca}_{0}=$ contenido de calcio de la pastura, $\mathrm{kg} / \mathrm{kg} \mathrm{MS}$.

Caj= contenido de calcio del j-esimo suplemento, $\mathrm{kg} / \mathrm{kg} \mathrm{MS}$.

$\mathrm{P}_{0}=$ contenido de fósforo de la pastura, $\mathrm{kg} / \mathrm{kg} \mathrm{MS}$.

$\mathrm{Pj}=$ contenido de fósforo del j-esimo suplemento, $\mathrm{kg} / \mathrm{kg} \mathrm{MS}$.

Req_Ca $=$ requerimientos de calcio para mantenimiento y crecimiento, $\mathrm{kg} / \mathrm{d}$ ía.

Req $\mathrm{P}=$ requerimientos de fósforo para mantenimiento y crecimiento, $\mathrm{kg} / \mathrm{día}$.

Req_MP= requerimientos de proteína metabolizable para mantenimiento y crecimiento, $\mathrm{kg} / \mathrm{d}$ ía.

$\mathrm{Km}_{\mathrm{i}}=$ eficiencia de utilización de la EM para mantenimiento para i-esima densidad calórica de la dieta. $\mathrm{Kc}_{\mathrm{i}}=$ eficiencia de utilización de la EM para crecimiento para i-esima densidad calórica de la dieta. $\mathrm{dEM}_{\mathrm{i}}=\mathrm{i}$-esima densidad calórica de la dieta (Mcal EM/kg MS).

$M=$ constante numérica suficientemente grande, ej. 999.

$\mathrm{NEm}=$ requerimientos de EN para mantenimiento, Mcal/día.

$\mathrm{RE}=$ requerimientos de EN para crecimiento, Mcal/día.

NEmact= requerimientos de ENm por la actividad física del pastoreo, Mcal/día.

$\mathrm{NEmhs}=$ requerimientos de ENm por estrés por calor, Mcal/día.

NEmcs= requerimientos de ENm por estrés por frío, Mcal/día.

$\mathrm{Tc}=$ temperatura ambiente actual, ${ }^{\circ} \mathrm{C}$.

$\mathrm{SA}=$ área de la superficie corporal, $\mathrm{m}^{2}$.

$\mathrm{IN}=$ valor de aislamiento, ${ }^{\circ} \mathrm{C} / \mathrm{Mcal} / \mathrm{m}^{2} /$ día.

$\mathrm{CMS}_{\mathrm{i}}=$ consumo potencial de materia seca para la i-esima densidad energética de la dieta.

$\mathrm{SBW}=$ peso vivo desbastado del animal, $\mathrm{kg}$.

$\mathrm{BI}=$ factor de corrección del consumo por efecto de la raza del animal.

$\mathrm{BFAF}=$ factor de corrección del consumo por efecto del grado de engrasamiento del animal.

$\mathrm{ADTV}=$ factor de corrección del consumo por efecto del empleo de estimulantes del crecimiento.

TEMP1 = factor de corrección del consumo por efecto de la temperatura ambiente.

MUD1= factor de corrección del consumo por efecto de la presencia de barro.

EfSint= eficiencia de síntesis de proteína microbiana, $\mathrm{kg} / \mathrm{kg}$ TDN.

consumo en función de la densidad energética de la dieta.

\section{Consumo de nutrientes}

El consumo total de EM(MEI), nutrientes digestibles totales (TDN_cons), proteína degradable (DIP cons), proteína indegradable (UIP_cons), fibra neutro detergente efectiva (eNDF_cons), calcio (Ca_cons) y fósforo ( $\mathrm{P}$ cons $\bar{s}$ son computados porque posteriormente algunos de ellos determinan los requerimientos del animal en el

Archivos de zootecnia vol. 61, núm. 234, p. 258. 
modelo nivel uno del NRC (2000).

$$
\begin{aligned}
& \text { R5) } \mathrm{PS}^{*} \mathrm{EM}_{0}+\Sigma \mathrm{X}_{\mathrm{j}}{ }^{*} \mathrm{EM}_{\mathrm{j}}-\mathrm{MEI}=0 \\
& \text { R6) } P s^{*} \operatorname{TDN}_{0}+\Sigma X_{j}^{*} \operatorname{TDN}_{j} \quad-T D N_{-} \text {cons }=0 \\
& \text { R7) } P_{S}{ }^{*} U_{I}+\sum X_{0}{ }^{*} U I P_{1} \quad-U I P_{-} \text {cons }=0 \\
& \text { R8) } P_{s}{ }^{*} \mathrm{DIP}_{0}+\sum \mathrm{X}_{\mathrm{j}}{ }^{*} \mathrm{DIP}_{\mathrm{j}}{ }^{\mathrm{j}} \quad-\mathrm{DIP}_{-} \text {cons }=0 \\
& \text { R9) } P s^{*} \text { eNDF }_{0}+\Sigma X_{j}{ }^{*} \text { eNDF }_{j} \text {-eNDF_cons }=0 \\
& \mathrm{R} 10) \mathrm{Ps}{ }^{*} \mathrm{Ca}_{0}+\Sigma \mathrm{X}_{\mathrm{j}}{ }^{*} \mathrm{Ca}_{\mathrm{j}} \quad-\mathrm{Ca}_{-} \text {cons }=0 \\
& \text { R11) } P s{ }^{*} P_{0}+\Sigma X_{j}{ }^{*} P_{j} \quad-P_{-} \text {cons } \quad=0
\end{aligned}
$$

\section{Calcio y fósforo}

El modelo considera los requerimientos de calcio y fósforo y el máximo consumo tolerado, los cuales se relacionan con el peso vivo del animal, la proteína retenida en la ganancia y el consumo de materia seca.

$$
\begin{array}{ll}
\text { R12) Ca_cons } & \geq \text { Req_Ca } \\
\text { R13) Ca_cons } & - \text { DMI * } 0,02 \leq 0 \\
\text { R14) P_cons } & \geq \text { Req_P } \\
\text { R15) P_cons } & - \text { DMI }{ }^{*} 0,01 \leq 0
\end{array}
$$

Donde Req_Ca y Req_P son los requerimientos para mantenimiento y crecimiento de calcio y fósforo respectivamente, calculados de acuerdo a las especificaciones de la tabla 10-2 (pág. 118) del NRC (2000).

\section{Proteína metabolizable}

El NRC (2000) expresa los requerimientos proteicos del animal en proteína metabolizable(MP), la cual es definida como la proteína verdadera absorbida a nivel intestinal, cuya fuente son las porciones digestibles de la proteína microbiana y de la proteína no degradable en rumen de la dieta. El sistema de MP adoptado por el NRC (2000) se basa en unos cuantos supuestos. La MP aportada por la dieta (MPfeed) viene determinada por la proteína no degradable de la dieta(UIP) multiplicado por 0,80 ya que se asume una digestibilidad de la misma del $80 \%$. La MP aportada por los microorganismos del rumen (MPbact) corresponde a la producción de proteína bruta microbiana (MCP) multiplicada por 0,64 (80\% es proteína verdadera con una digestibilidad del $80 \%$ ). La producción de MCP esta determinada por el factor más limitante, el consumo de energía (TDN_cons) o el consumo de proteína degradable en rumen proveniente de la dieta(DIP_cons). El NRC (2000) computa la producción de MCP en base al consumo de energía y en consecuencia establece que el consumo de DIP debe ser al menos suficiente para cubrir las necesidades de nitrógeno de los microorganismos del rumen. Para la síntesis de MCP a partir de la DIP el NRC (2000) considera una eficiencia de 1:1 ya que asume que la pérdida de amoníaco del rumen por pasaje al duodeno y por absorción a través de la pared ruminal es igual a la cantidad reciclada de nitrógeno. Mientras que para la síntesis de MCP a partir de la energía (EfSint), el NRC (2000) asume una producción estándar de $130 \mathrm{~g}$ de MCP por kg de TND ingerido, para dietas con NDF efectivo mayor al 20\%. Existen evidencias experimentales que sugieren que rumiantes alimentados con forrajes de mediana a baja calidad presentarían una eficiencia de síntesis de MCP menor a la estándar (NRC, 2000), siendo del orden de los 80 a $110 \mathrm{~g} / \mathrm{kg}$ TND dependiendo de la calidad del forraje (Lardy et al., 2004; DelCurto etal., 2000; Cochran et al., 1998).

$\begin{array}{llc}\text { R16) MPbact } & + \text { MPfeed } & \geq \text { Req_MP } \\ \text { R17) UIP_cons }{ }^{*} 0,80 & - \text { MPfeed } & =0 \\ \text { R18) MCP } 0,64 & - \text { MPbact } & =0 \\ \text { R19) DIP_cons } & - \text { MCP } & \geq 0 \\ \text { R20) TDN_cons EfSint } & - \text { MCP } & =0 \\ \text { R21) eNDF_cons } & -0,20 \text { DMI } & \geq 0\end{array}$

Donde Req_MP son los requerimientos de proteína metabolizable del animal para mantenimiento y crecimiento calculados mediante las ecuaciones habituales del NRC (2000, pág. 116), las cuales tienen en cuenta el peso vivo del animal, la ganancia diaria, la composición corporal, el peso corporal relativo y la densidad calórica de la ganancia.

\section{Energía}

La EM es la diferencia entre el calor liberado en la combustión completa del alimento (energía bruta) y la energía perdida en heces, orina y gases (NRC, 2000).

La energía neta $(\mathrm{EN})$ es la diferencia 


\section{SOTOYREINOSO}

entre la EM y el incremento calórico (pérdida de calor) que se produce en el organismo debido a los procesos de digestión y metabolización del alimento (NRC, 2000).

Los requerimientos energéticos del animal para mantenimiento y crecimiento expresados en EN son totalmente independientes de la dieta (NRC, 2000), en cambio los requerimientos expresados en energía metabolizable dependen de la eficiencia de utilización de la misma ya que parte de la EM consumida se pierde por el incremento calórico ocasionado por los procesos de la digestión(NRC, 2000). Si bien el sistema de EN es totalmente independiente de la dieta, al ser la EN para mantenimiento (ENm) y crecimiento (ENg) empleada con diferente eficiencia, el sistema de energía neta es no aditivo (Lofgreen y Garrett, 1968).

Existen diferentes procedimientos (Brokken, 1971; Harkins et al., 1974) para tratar la no aditividad del sistema de EN. Los procedimientos diseñados por los autores antes mencionados asumen que los requerimientos totales de EN de mantenimiento y crecimiento para un determinado nivel de producción son constantes conocidas al momento de formular el modelo, lo cual en el caso del NRC (2000) es verdadero para animales en ambientes termoneutros y en ambientes con estrés por calor. En cambio el incremento de los requerimientos de mantenimiento debido al estrés por frío propuesto por el NRC (2000) es totalmente dependiente de la dieta consumida, lo cual hace necesario la implementación de un procedimiento alternativo para tratar este caso especial de falta de aditividad.

El estrés por calor o por frío se produce cuando la temperatura ambiente es superior o inferior respectivamente al rango de temperatura de la zona termoneutra (NRC, 2000). Tanto el estrés por frío como por calor exigen del animal una serie de cambios conductuales y fisiológicos para tratar de mantener constante la temperatura corporal, estos cambios exigen energía adicional y por lo tanto incrementan las necesidades de mantenimiento (NRC, 2000).

El consumo de EM (MEI) que realiza el animal debe cubrir sus requerimientos (EM_req), los cuales para animales en crecimiento-engorde a pastoreo son la sumatoria de los requerimientos de EM para mantenimiento (EMm), crecimiento-engorde $(\mathrm{EMg})$, actividad física del pastoreo (EMmact) y estrés por calor (EMhs) o por frío (EMcs) caso existan.

R22) $M E I$ - EM_req $\geq 0$

R23) $E M m+E M g$ + EMmact + EMhs + EMcs - EM_req $=0$

Los requerimientos de EM para los cuatro primeros procesos mencionados (EMm, EMg, EMmact, EMhs) se obtienen determinando previamente los correspondientes requerimientos de energía neta (NEm, RE, NEmact, NEmhs respectivamente) de acuerdo a las ecuaciones presentadas por el NRC (2000, pág. 114 a 116) los cuales son convertidos por el modelo de PLEM a EM según la eficiencia de utilización de la energía $(\mathrm{km}$, $\mathrm{kc})$ que presente la dieta final formulada. Los requerimientos por estrés por frío se obtienen directamente en EM de acuerdo a las ecuaciones del NRC (2000, pág. 114 y 115).

Como la eficiencia de utilización de la EM para mantenimiento $(\mathrm{km})$ y crecimiento (kc) dependen de la densidad calórica de la dieta y esta a su vez depende de la relación entre la EM consumida y el consumo total de materia seca (EM [Mcal/kg MS]= MEI / DMI) (NRC, 2000) los cuales no se conocen a priori, sino que son variables a determinar en el modelo, esto torna evidentemente al sistema en no aditivo.

Existen diferentes maneras de incorporar funciones no lineales y no aditivas a modelos de PL, los cuales han sido descritos con detalle en numerosos trabajos (Hiller y Lieberman, 2002; Taha, 1998; Apland ,1986; Black y Hlubick, 1980), inclusive algunos han sido aplicados específicamente a la formulación de raciones (Tedeschi et al., 2000; Garcia Martinez et al., 1998; Brokken, 1971; 
FORMULACIÓN DE RACIONES BASADO EN EL SISTEMA NRC 2000

Dean et al., 1969). En el presente trabajo la falta de aditividad y linealidad de algunas funciones del Modelo Nivel Uno del NRC (2000) fueron superadas mediante el empleo de restricciones alternativas modeladas con variables binarias (Baíllo et al., 2004; Hiller y Lieberman, 2002), el caso general de las restricciones alternativas son un conjunto de $\mathrm{N}$ restricciones de las cuales han de satisfacerse $\mathrm{K}$ de ellas, siendo $\mathrm{K}<\mathrm{N}$ :

$$
\begin{aligned}
& \sum a_{1 j}{ }^{*} X_{j} \leq b_{1} \\
& \sum a_{2 j}{ }^{*} X_{j} \leq b_{2} \\
& \ldots \ldots \ldots \ldots \ldots \ldots \ldots \\
& \sum a_{n j}{ }^{*} X_{j} \leq b_{n}
\end{aligned}
$$

Añadiendo apropiadamente a cada restricción una constante M lo suficientemente grande $y$ una variable binaria $y_{i}$ se obtiene:

$$
\begin{aligned}
& \sum a_{1 j}{ }^{*} X_{j} \leq b_{1}+M\left(1-y_{1}\right) \\
& \sum a_{2 j}{ }^{*} X_{j} \leq b_{2}+M\left(1-y_{2}\right) \\
& \sum a_{n j}{ }^{*} X_{j} \leq b_{n}+M\left(1-y_{n}\right)
\end{aligned}
$$

dónde además se impone la condición de seleccionar solamente $\mathrm{K}$ restricciones

$$
\sum y_{i}=K
$$

de manera que si $y_{i}=1$ la restricción iesima se activa ( se cumple), si $y_{i}=0$ la $i$-esima restricción se relaja (es redundante) (Baíllo et al., 2004; Hiller y Lieberman, 2002).

Las restricciones del tipo mayor igual se modelan como:

$$
\Sigma a_{i j}{ }^{*} X_{j} \geq b_{i}-M\left(1-y_{i}\right)
$$

Mientras que las restricciones alternativas del tipo igual se modelan descomponiéndolas en dos restricciones equivalentes de menor igual y mayor igual:

$$
\begin{aligned}
& \sum a_{i j}{ }^{*} X_{j} \leq b_{i}+M\left(1-y_{j}\right) \\
& \sum a_{i j}{ }^{*} X_{j} \geq b_{i}-M\left(1-y_{i}\right)
\end{aligned}
$$

En la práctica, el valor de $\mathrm{M}$ debe ser suficientemente grande para actuar como una penalidad, pero no debe ser tan grande que desequilibre la exactitud de los cálculos debido a errores de redondeo del ordenador, lo cual puede ocurrir al manipular una mezcla de números grandes y pequeños.

El modelo presentado en este trabajo parametriza la densidad calórica de la dieta en N valores de Mcal EM/ kg MS, como la densidad energética es no aditiva (depende de la relación consumo de energía/consumo

\begin{tabular}{|c|c|c|c|c|}
\hline R24.1a) & MEI & $-\mathrm{DMI}^{*} \mathrm{dEM}_{1}$ & $+y_{1}{ }^{*} M$ & $\leq M$ \\
\hline$R 24.1 b)$ & ME I & $-\mathrm{DMI}^{*} \mathrm{dEM}_{1}$ & $-y_{1}{ }^{*} M$ & $\geq-M$ \\
\hline R24.2a) & ME I & $-\mathrm{DMI}^{*} \mathrm{dEM}_{2}$ & $+y_{2} * M$ & $\mathrm{M}$ \\
\hline $\mathrm{R} 24.2 \mathrm{~b})$ & MEI & - DMI * dEM & $-y_{2}{ }^{*} M$ & $\geq-M$ \\
\hline R24.Na) & MEI & $-\mathrm{DMI}^{*} \mathrm{dEM}$ & $+y_{n} * M$ & $\leq M$ \\
\hline R24.Nb) & MEI & - DMI * dEM & $-y_{n}{ }^{*} M$ & $\geq-M$ \\
\hline 25) & & & & \\
\hline
\end{tabular}
de alimentos) se establecen $\mathrm{N}$ restricciones alternativas para los posibles $\mathrm{N}$ valores de $\mathrm{EM} / \mathrm{kg} \mathrm{MS}$ de la dieta, imponiendo la condición adicional que debe satisfacerse sólo una de las $\mathrm{N}$ restricciones alternativas, de manera que el modelo selecciona la densidad calórica más conveniente, la que respetando las restricciones nutricionales impuestas minimiza el costo total de la dieta.

En el ejemplo del presente trabajo se hizo arbitrariamente $\mathrm{dEM}_{1}=1,40 \mathrm{Mcal} \mathrm{EM} / \mathrm{kg} \mathrm{MS}$ $\mathrm{y} \mathrm{dEM}_{\mathrm{n}}=3,50 \mathrm{Mcal} \mathrm{EM} / \mathrm{kg} \mathrm{MS}$, con intervalos de $0,05 \mathrm{Mcal}$, totalizando $\mathrm{n}=43$ posibles densidades energéticas de la dieta $\left(\mathrm{dEM}_{\mathrm{i}}\right)$. Conociendo la densidad calórica de la dieta $\left(\mathrm{dEM}_{\mathrm{i}}\right)$ se obtienen los correspondientes valores de $\mathrm{Km}$, Kc y el consumo potencial de materia seca tal como se describe en el NRC (2000), por ejemplo una dieta con 2,50 Mcal EM/kg MS posee un $\mathrm{Km}=$ 0,643 , un $\mathrm{Kc}=0,401$ y un consumo potencial en animales en crecimiento-engorde de $0,1145 \mathrm{~kg} \mathrm{MS} / \mathrm{kg} \mathrm{SBW}^{0,75}$.

Conociendo la eficiencia de utilización de la energía para cada posible densidad energética de la dieta, los requerimientos 


\section{SOTOYREINOSO}

expresados en EM se modelan empleando restricciones con $\mathrm{N}$ posibles valores alternativos del lado derecho, donde el caso general se escribe como (Hiller y Lieberman, 2002):

$$
\begin{aligned}
& \sum a_{n j}{ }^{*} X_{j}=\sum b_{i}{ }^{*} y_{i} \\
& \sum y_{i}=1
\end{aligned}
$$

de manera que si la i-esima variable binaria $y_{i}=1$ se satisface el correspondiente i-esimo valor del lado derecho $\left(b_{i}\right)$.

R26) $\mathrm{y}_{1}{ }^{*}\left(\mathrm{NEm} / \mathrm{Km}_{1}\right)+\ldots+\mathrm{y}_{\mathrm{N}}{ }^{*}\left(\mathrm{NEm} / \mathrm{Km}_{\mathrm{n}}\right) \quad-\mathrm{EMm}=0$ R27) $y_{1}{ }^{*}\left(R E g / K c_{1}\right) \quad+\ldots+y_{N}^{*}\left(R E g / K c_{n}\right) \quad-E M g=0$ R28) $y_{1}{ }^{*}\left(N E m a c t / K m_{1}\right)+\ldots+y_{N}{ }^{*}\left(N E m a c t / K m_{n}\right)-E M m a c t=0$ R29) $y_{1}{ }^{*}\left(N E m h s / K_{1}\right)+\ldots+y_{N}{ }^{*}\left(N E m h s / K m_{n}\right)-E M h s=0$

Al ser de las $\mathrm{N}$ variables binarias solo una la activada a nivel 1 (la correspondiente a la densidad calórica de la dieta seleccionada) como lo impone la restricción R25, el modelo computa como requerimientos energéticos expresados en EM sólo aquellos valores que corresponden a la variable y activada, ignorando los demás valores pues sus correspondientes variables binarias se encontrarán a nivel cero.

Integrando las ecuaciones del NRC (2000, pág. 114 y 115) la restricción con los requerimientos en EM por estrés por frío se escribe como:

R30) MEcs + MEI * $0,85 \geq\left[(39-T c)^{*} S A / I N+0,85\right.$ * RE $]$

donde Tc, SA, IN y RE se determinan como lo indica el NRC (2000, pág. 114 y 115).

\section{Consumo voluntario potencial}

El consumo potencial de materia seca en animales en crecimiento-engorde es estimado por el NRC (2000, pág. 118 y 119) mediante una función no lineal que tiene en cuenta la densidad energética de la dieta y el peso vivo del animal, ajustado según la raza (BI), el grado de engrasamiento (BFAF), el empleo de estimulantes del crecimiento (ADTV), la temperatura ambiente (TEMP1) y la presencia de barro (MUD1).
R31) $\Sigma y_{i}{ }^{*}\left(\mathrm{CMS}_{\mathrm{i}}{ }^{*} \mathrm{SBW} \mathrm{W}^{0,75}{ }^{*} \mathrm{BI}{ }^{*} \mathrm{BFAF}{ }^{*} \mathrm{ADTV}\right.$ *TEMP1 ${ }^{*}$ MUD1) - DMI $\geq 0$

donde:

CMS es el consumo potencial de materia seca por kg de peso metabólico para la i-esima densidad energética de la dieta,

BI, BFAF, ADTV, TEMP1, MUD1 son factores multiplicativos extraídos de la tabla 10-4 del NRC (2000, pág. 119) que ajustan el consumo por los efectos antes mencionados.

\section{Ampliación del modelo}

El modelo descrito anteriormente considera constante la eficiencia de síntesis (EfSint) de MCP a partir de la energía (R20), y para ello requiere que el contenido de eNDF de la dieta sea como mínimo del $20 \%$ (R21). Existe una estrecha relación entre la concentración de eNDF y el pH ruminal, el NRC (2000) establece que cuando el eNDF desciende por debajo del $20 \%$ de la dieta la eficiencia de síntesis de la proteína microbiana disminuye un $2,5 \%$ por cada $1 \%$ de disminución del eNDF debido a la concomitante reducción del $\mathrm{pH}$ ruminal. Para considerar este hecho las restricciones R20 y R21 se sustituyen por restricciones alternativas que parametrizan el contenido de eNDF y la correspondiente eficiencia de síntesis microbiana. Se estableció un mínimo de 5\% de eNDF en la dieta para mantener

\begin{tabular}{|c|c|c|c|}
\hline R21.1) & eNDF_cons & $-\mathrm{DMI} * 0,20$ & $-\rho_{1}{ }^{*} \mathrm{M} \geq-\mathrm{M}$ \\
\hline $\mathrm{R} 21.2 \mathrm{a})$ & eNDF_cons & $-\mathrm{DMI} * 0,1999$ & $+\rho_{2}{ }^{*} M \leq M$ \\
\hline $\mathrm{R} 21.2 \mathrm{~b})$ & eNDF_cons & - DMI * 0,1900 & $-\rho_{2}{ }^{*} M \geq-M$ \\
\hline R21.16a) & eNDF_cons & $-\mathrm{DMI}^{*} 0,0599$ & $+\rho_{16}{ }^{*} \mathrm{M} \leq \mathrm{M}$ \\
\hline $\mathrm{R} 21.16 \mathrm{~b})$ & eNDF_cons & - DMI *0,0500 & $-\rho_{16}{ }^{*} M \geq-M$ \\
\hline R21.0) & & $\Sigma \rho_{\mathrm{i}}=1$ & \\
\hline $\mathrm{R} 20.1 \mathrm{a})$ & TDN_cons ${ }^{*} \mathrm{E}$ & $f$ Sint $^{* 1,00}-\mathrm{MCP}$ & $+\rho_{1}{ }^{*} M \leq M$ \\
\hline $\mathrm{R} 20.1 \mathrm{~b})$ & TDN_cons ${ }^{*} \mathrm{E}$ & fSint*1,00-MCP & $-\rho_{1}{ }^{*} M \geq-M$ \\
\hline $\mathrm{R} 20.2 \mathrm{a})$ & TDN_cons ${ }^{*} \mathrm{E}$ & EfSint ${ }^{\star} 0,975-\mathrm{MCP}$ & $+\rho_{2}{ }^{*} M \leq M$ \\
\hline$R 20.2 b)$ & TDN_cons ${ }^{*} \mathrm{E}$ & EfSint ${ }^{*} 0,975-\mathrm{MCP}$ & $-\rho_{2}{ }^{*} M \geq-M$ \\
\hline
\end{tabular}
una adecuada salud ruminal (NRC, 2000).

R20.16a) TDN_cons ${ }^{*} E f S i n{ }^{*} 0,625-\mathrm{MCP}+\rho_{16}{ }^{*} \mathrm{M} \leq \mathrm{M}$ R20.16b) TDN_cons ${ }^{\star} E f S i n t{ }^{*} 0,625-\mathrm{MCP}-\rho_{16}{ }^{*} \mathrm{M} \geq-\mathrm{M}$

El modelo selecciona la concentración de eNDF de la dieta más conveniente y 
FORMULACIÓN DE RACIONES BASADO EN EL SISTEMA NRC 2000

Tabla III. Descripción de las características del animal y supuestos ambientales. (Description of animal and environmental characteristics).

\begin{tabular}{llc}
\hline Descripción & Variable & Valor \\
\hline Sexo & & novillitos \\
Raza & Hereford \\
Edad (meses) & 9 \\
Peso vivo desbastado (kg) & TE & 160 \\
Ganancia diaria (kg/día) & SBW & 0,200 \\
Condición corporal (1 a 9) & SWG & 5 \\
Peso de referencia para engrasamiento deseado (kg) & CS & 462 \\
Disponibilidad inicial de pastura (kg MS/ha) & SRW & 800 \\
Ondulación del terreno & pAVAIL, IPM & leve \\
TDN de la pastura (\%) & TERRAIN \\
Tiempo de pastoreo (días) & TDNp \\
Superficie de pastoreo (ha) & DOP & 90 \\
Numero de animales (cabezas) & GU & 1 \\
Velocidad del viento (Kph) & N & 1 \\
Temperatura previa ( ${ }^{\circ}$ C) & WIND & 15 \\
Temperatura actual ( $\left.{ }^{\circ} \mathrm{C}\right)$ & Tp & 7 \\
Noches frescas & TC & 7 \\
Largo del pelo (cm) & TEMP1 & Si \\
Tipo de cuero & HAIR & 0,5 \\
Capa de pelo & HIDE & grueso \\
Estrés por calor & MU2 & no \\
Ef. síntesis proteína microbiana (kg/kg TDN) & -130 \\
\hline
\end{tabular}

mediante la correspondiente variable binaria $\rho_{i}$ activada asigna la eficiencia de síntesis microbiana adecuada.

\section{Ejemplo de aplicación del modelo}

A partir de los datos de las tablas III y IV se desarrolló un ejemplo hipotético de aplicación del modelo para novillitos a pastoreo durante el periodo invernal. Por el espacio, la matriz de programación lineal que contiene el planteo del ejemplo no se incluyó, puede solicitarse por correo electrónico.

Tabla IV. Composición de los alimentos disponibles (adaptado del NRC, 2000 y Mieres, 2004). (Composition of available feed (adapted from NRC 2000 and Mieres 2004)).

\begin{tabular}{lcccc}
\hline & Campo natural & Maíz quebrado & Expeller girasol & Afrechillo arroz \\
\hline Costo (U\$S/kg MS) & 0,007 & 0,145 & 0,133 & 0,105 \\
eNDF $(\mathrm{kg} / \mathrm{kg} \mathrm{MS})$ & 0,225 & 0,065 & 0,092 & 0,000 \\
TDN $(\mathrm{kg} / \mathrm{kg} \mathrm{MS})$ & 0,550 & 0,900 & 0,650 & 0,700 \\
EM (Mcal/kg MS) & 1,980 & 3,250 & 2,350 & 2,530 \\
PB (kg/kg MS) & 0,0915 & 0,098 & 0,259 & 0,144 \\
DIP $(\mathrm{kg} / \mathrm{kg} \mathrm{MS})$ & 0,0595 & 0,044 & 0,207 & 0,073 \\
UIP $(\mathrm{kg} / \mathrm{kg} \mathrm{MS})$ & 0,0320 & 0,054 & 0,052 & 0,071 \\
Ca $(\mathrm{kg} / \mathrm{kg} \mathrm{MS})$ & 0,0038 & 0,0003 & 0,0045 & 0,0010 \\
P $(\mathrm{kg} / \mathrm{kg} \mathrm{MS})$ & 0,0014 & 0,0032 & 0,0102 & 0,0173 \\
\hline
\end{tabular}




\section{SOTOYREINOSO}

\section{RESULTADOSYDISCUSIÓN}

El presente trabajo describe como incorporar el Modelo Nivel Uno del NRC (2000) a un modelo de programación lineal entero mix to para formular dietas de mínimo costo para ganado de carne a pastoreo. El modelo explora aquella combinación de alimentos que arroje el menor costo y a su vez cubra los requerimientos de energía, proteína metabolizable, calcio, fósforo y fibra efectiva para las funciones de mantenimiento $y$ crecimiento de los animales.

El campo natural es la principal base forrajera de la ganadería de carne en Uruguay, el mismo se caracteriza por poseer una pastura de mediana a baja calidad (2,0 a 2,2 Mcal EM/kg MS, 9 a 12\% PB) y una marcada fluctuación en la producción de forraje. Generalmente se observa una baja producción de forraje en invierno y un superávit en primavera-verano (Orcasberro, 1994; Mieres, 2004). La recría (terneros destetados y sobreaños) suele pastorear exclusivamente campo natural sin ningún tipo de alimentación suplementaria, debido principalmente a la baja disponibilidad forrajera del campo natural durante el invierno suelen observarse pérdidas de hasta 10 a $15 \%$ del peso vivo en estas categorías, lo cual impide alcanzar un ritmo de crecimiento adecuado para llegar con peso y estado suficientes para un apareamiento exitoso a las 2 años de edad en las hembras y un peso adecuado de faena a los 2,5 años de edad en los machos (Quintans, 2002; Pigurina et al., 1997).

La investigación ha sido consistente en confirmar que para lograr los objetivos antes mencionados de apareamiento y faena a edades relativamente tempranas es necesario que la recría durante su primer y segundo invierno obtengan ganancias moderadas de peso (aproximadamente $0,200 \mathrm{~kg} /$ día) y para ello se requiere una suplementación con concentrados durante el período invernal de entre 0,7 al $1 \%$ del peso vivo de los animales (Quintans, 2002; Pigurina et al., 1997).
El ejemplo planteado no pretende ser una recomendación, simplemente ilustra la implementación del modelo de PLEM, si bien el mismo es hipotético intenta representar una situación bastante frecuente en la ganadería de carne extensiva de Uruguay simulando las condiciones pastoriles y ambientales de los terneros luego del destete en su primer invierno y utilizando como posibles suplementos alimentos típicos de esta región.

Para que terneros de $160 \mathrm{~kg}$ de PV pastoreando campo natural de baja disponibilidad puedan obtener ganancias de 0,200 $\mathrm{kg}$ /día, la salida del modelo de PLEM arroja una dieta compuesta por $76 \%$ de pastura (3,33 kg MS/animal/día) y $24 \%$ de concentrados $(0,2922 \mathrm{~kg} \mathrm{MS} /$ animal/día de expeller de girasol más $0,7594 \mathrm{~kg} \mathrm{MS} /$ animal/día de afrechillo de arroz). Asumiendo un contenido promedio de MS de $88 \%$ en los concentrados el modelo estima un nivel de suplementación de $0,75 \%$ del peso vivo lo cual concuerda con las recomendaciones de las investigaciones al respecto (Quintans, 2002; Pigurina et al., 1997). El suplemento así formulado está integrado por $27,8 \%$ de expeller de girasol y $72,2 \%$ de afrechillo de arroz lo cual aportaría 2,5 Mcal EM/kg MS y $17,6 \%$ de $\mathrm{PB}$, este contenido de nutrientes coincide con los concentrados usualmente recomendados por los nutricionistas para la categoría animal y condiciones ambientales descritas en el ejemplo (2,4 a 2,8 Mcal EM/ kg MS, 16 a $18 \%$ PB).

Por otro lado, Lardy et al. (2004) revisando el modelo nivel uno del NRC para vacas de cría a pastoreo concluyeron que el modelo es un buen predictor de las necesidades de suplemento, pero requiere una serie de ajustes en los datos de entrada, fundamentalmente los relacionados al ambiente y al manejo para que pueda brindar salidas consistentes con la realidad pastoril.

El modelo PLEM presentado en este trabajo puede ser fácilmente ampliado, se puede restringir el uso de determinados suplementos, imponer determinadas relaciones 
entre algunos alimentos, establecer requerimientos de otros minerales y de vitaminas A y D, etc. El modelo puede ser aplicado a vacas de cría, simplemente incorporando restricciones que contemplen los requerimientos energéticos para lactación y gestación, sumar a los requerimientos totales de $\mathrm{PM}$ los requerimientos de estos dos procesos y parametrizar el consumo potencial de materia seca en función de la densidad energética de la dieta con la ecuación descrita por el NRC (2000) para dicha categoría. Los requerimientos energéticos para gestación se pueden obtener directamente en energía metabolizable, mientras que los de lactación se parametrizan de manera similar como se hace con las necesidades de mantenimiento en la restricción R26 dado que la EM se emplea con similar eficiencia para mantenimiento y para lactación (NRC 2000). Las dos nuevas variables que contemplen las necesidades de EM para gestación y lactación se suman a la de los requerimientos totales (R23). El modelo también puede ser fácilmente adaptado para formular dietas para

\section{BIBLIOGRAFÍA}

Apland, J. 1986. The approximation of nonlinear programming problems using linear programming. Department of Agricultural and Applied Economics. University of Minnesota. Staff Paper P86-2. 23 pp.

Baíllo, A., Linares, P., Ramos, A., Sánchez, P., Sarabia, A. y Vitoriano, B. 2004. Modelos matemáticos de optimización. Escuela Técnica Superior de Ingeniería. Universidad Pontificia Comillas. Madrid. 199 pp.

Bargo, F., Muller, L., Kolver, E. and Delahoy. 2003. Invited review: Production and digestion of supplemented dairy cows on pasture. J Dairy Sci, 86: 1-42.

Barnard, C. y Nix, J. 1984. Planeamiento y control agropecuario. Ed. El Ateneo. Buenos Aires. 527 pp.

Beneke, R. and Winterboer, R. 1973. Linear Programming Applications to Agriculture. The lowa State University Press. 244 pp.

Black, J. and Hlubik, J. 1980. Basics of computerized animales en condiciones de engorde a corral (feed-lot), simplemente eliminando del modelo los parámetros referentes al pastoreo (haciendo $\mathrm{pI}=0$ y EMmact $=0$ en las restricciones R2 y R23 respectivamente).

La complejidad de los modelos del NRC (2000) requieren modelos para formular raciones al mínimo costo más complejos que las versiones anteriores. El modelo de PLEM descrito en este trabajo presenta un grado de complejidad intermedia (180 restricciones y 84 variables de decisión de las cuales 59 son binarias), el planteo y solución con programas informáticos que soporten lenguajes lexicográficos de programación matemática facilitan enormemente el mantenimiento y actualización del modelo, minimizando la posibilidad de errores involuntarios en la introducción y modificación de datos.

\section{AGRADECIMIENTOS}

Al Dr. Pablo Lara (Departamento de Producción Animal, Universidad de Córdoba, España) por la lectura del manuscrito y por sus valiosas sugerencias.

linear programs for ration formulation. $J$ Anim Sci, 63: 1366-1378.

Brokken, R. 1971. Programming models for use of the Lofgreen-Garrett net energy system in formulating rations for beef cattle. J Anim Sci, 32: 685-691.

Caton, J. and Dhuyvetter, D. 1997. Influence of energy supplementation on grazing ruminants: Requirements and responses. J Anim Sci, 75: 533-542.

Cochran, R., Koster, H., Olson, K., Heldt, J., Mathis, C. and Woods, B. 1998. Supplemental protein sources for grazing beef cattle, Proc. $9^{\text {th }}$ Annual Florida Ruminant Nutrition Symposium. University of Florida. Gainesville.

Dean, G., Bath, D. and Olayide, S. 1969. Computer program for maximizing income above feed cost from dairy cattle. J Dairy Sci, 52: 1008-1016.

DelCurto, T., Hess, B., Huston, J. and Olson, K.C. 2000. Optimum supplementation strategies for beef cattle consuming low-quality roughages in 


\section{SOTOYREINOSO}

the western United States. Proc Am Soc Anim Sci, 1999.

Dixon, R. and Stockdale, C. 1999. Associative effects between forages and grains: consequences for feed utilization. Aust J Agric Res, 50: 757-773.

Garcia Martinez, A., Rodriguez Alcaide, J. y Ruiz, D. 1998.Optimización del engorde de bovinos en pastoreo en la pampa Argentina mediante programación lineal. Invest Agr: Prod Sanid Anim, 13: 99-117.

Harkins, J., Edwards, R. and McDonald, P. 1974 A new net energy system for ruminants. Anim Prod, 19: 141-148.

Hiller, F. y Lieberman, G. 2002. Investigación de operaciones. $7^{a}$ ed. McGraw Hill. México. 1223 $\mathrm{pp}$

Lardy, G., Adams, D., Klopfenstein, T. and Patterson, H. 2004. Building beef cow nutritional programs with the 1996 NRC beef cattle requirements model. J Anim Sci 82 (E. Suppl.): E83-E92.

Lofgreen, G. and Garrett, W. 1968. A system for expressing net energy requirements and feed values for growing and finishing cattle. J Anim Sci, 27: 739-806.

Maroto, C., Ciria, J., Gallego, L. y Torres, A. 1997. Gestión de la producción ganadera. Ediciones Mundi-Prensa. Madrid. 238 pp.

McCall, D., Clark, D., Stachurski, L., Penno. J., Bryant, A. and Ridler, B. 1999. Optimized dairy grazing systems in the northeast United States and New Zealand. I. Model description and evaluation. J Dairy Sci, 82: 1795-1807.

Mieres, J. 2004. Guía para la alimentación de rumiantes. INIA Serie Técnica, n 142. 81 pp.

Moore, J., Brant, M., Kunkle, W. and Hopkins, D. 1999. Effects of supplementation on voluntary forage intake, diet digestibility, and animal performance. J Anim Sci, 77 (Suppl. 2): 122-135.

NRC. 2000. Nutrient requirements of beef cattle, $7^{\text {th }}$ revised edition. Update 2000. National Academy Press. Washington, D.C. 248 pp.

O'Connor, J., Sniffen, C., Fox, D. and Milligan, R. 1989. Least cost dairy cattle ration formulation model based on the degradable protein system. J Dairy Sci, 72: 2733-2745.

Orcasberro, R. 1994. Suplementación y performance de ovinos y vacunos alimentados con forraje. En: Pasturas y producción animal en áreas de ganadería extensiva. INIA Serie Técnica, 13: 225-232.

Pigurina, G., Brito, G., Pittaluga, O., Scaglia, G., Risso, D. y Berretta, E. 1997. Suplementación de la recría en vacunos. En: Suplementación estratégica de la cría y recría ovina y vacuna. INIA Serie de Actividades de Difusión, 129: IV.1-IV.6.

Quintans, G. 2002. Manejo de la recría vacuna en sistemas ganaderos. Seminario de actualización técnica sobre la cría y recría ovina y vacuna. INIA Serie de Actividades de Difusión 288: 47-56.

Rotz, C., Mertens, D., Buckmaster, D., Allen, M. and Harrison, J. 1999. A dairy herd model for use in whole farm simulations. J Dairy Sci, 82: 28262840.

Soto, C. y Reinoso, V. 2004. Empleo de la programación lineal en la formulación de raciones al mínimo costo para la suplementación de rumiantes a pastoreo. Veterinaria, 39: 17-22.

Taha, H. 1998. Investigación de operaciones. Una introducción. 6a ed. Prentice Hall. México. 944 pp.

Tedeschi, L., Fox, D., Chase, L. and Wang, S. 2000. Whole-herd optimization with the Cornell net carbohydrate and protein system. I. Predicting feed biological values for diet optimization with linear programming. J Dairy Sci, 83: 2139-2148.

Waugh, F. 1951. The minimun cost dairy feed. An application of linear programming. J Farm Econ, 33: 299-310.

Archivos de zootecnia vol. 61, núm. 234, p. 266. 